\title{
Simulation-based Analysis of Ultra Thin-body Double Gate Ferroelectric TFET for an Enhanced Electric Performance
}

GIRDHAR GOPAL ( $\sim 2019$ rec9550@mnit.ac.in )

Malaviya National Institute of Technology https:// orcid.org/0000-0001-6212-6563

Tarun varma

MNIT Jaipur: Malaviya National Institute of Technology

\section{Research Article}

Keywords: BTBT, Memory Window, Subthreshold Swing, Threshold Voltage, UTB-DG-FE-TFET

Posted Date: August 5th, 2021

DOl: https://doi.org/10.21203/rs.3.rs-748137/v1

License: (c) (1) This work is licensed under a Creative Commons Attribution 4.0 International License.

Read Full License 


\title{
Simulation-based analysis of ultra thin-body double gate ferroelectric TFET for an enhanced electric performance
}

\author{
Girdhar Gopal · Tarun Varma
}

Received: date / Accepted: date

\begin{abstract}
The Ultrathin body double gate FE layer TFET(UTB-DG-FE-TFET) is proposed and investigated in this work. Electrical performance parameters such as surface potential $\psi(x)$, electrical field, drain current, sub-threshold swing, threshold voltage, and $I_{\text {on }} / I_{\text {off }}$ ratio are further analyzed using simulation-based analysis. Integration of $\mathrm{Si}: \mathrm{HFO}_{2}$ ferroelectric layer on top and bottom surfaces make the structure that provides negative capacitance, higher on current, enormous surface potential, peak electric field, and improvement in SS with degradation in off Current. The suggested design is evaluated in comparison with FE-TFET and standard TFET structures. Finally, the impact of device geometry variants like ferroelectric layer thickness $\left(t_{f e}\right)$, intrinsic channel thickness $t_{s i}$, interfacial layer types, interfacial layer thickness $\left(t_{o x}\right)$ and channel length $L c$ on transfer characteristics are investigated through 2D TCAD Sentaurus Simulator for a clear validation of its optimization. The recommended work demonstrates that it is a suitable device enabling superior performance and helpful in ultra-low-power applications.
\end{abstract}

Keywords BTBT · Memory Window · Subthreshold Swing · Threshold Voltage · UTB-DG-FE-TFET

Girdhar Gopal

Electronics and Communication Engineering Department, Malaviya National Institute of Technology Jaipur, Jaipur,Rajasthan 302017,India

E-mail: 2019rec9550@mnit.ac.in

Tarun Varma

Electronics and Communication Engineering Department, Malaviya National Institute of Technology Jaipur, Jaipur, Rajasthan 302017, India

\section{Introduction}

Short channel effects (SCEs) and an increase in leakage current are the main issues with downscaling the CMOS technology node [1-4]. Moreover, at room temperature $\mathrm{T}=300 \mathrm{~K}$, the scalability of threshold voltage $V_{T}$ is also limited due to Boltzmann tyranny ( $\mathrm{SS} \equiv$ $\frac{K T}{q} \cdot \ln 10 \approx 60 \mathrm{mV} /$ decade) that decides the steepest nature of the transition between on and off state [5$9]$. In short, there is a need for in-depth research on steep subthreshold swing devices $(<60 \mathrm{mV} /$ decade $)$ like Tunnel field-effect transistors(TFETs) [10,11], Impact ionization MOSFETs (I-MOS-FETs) [12], Nanoelectromechanical FETs (NEMFETs) [13] and Negative capacitance FETs [14]. These Emerging devices have been seriously explored in recent years due to impending physical constraints of conventional CMOS devices. Thereby TFETS or commonly called the green transistors, are the most promising contenders that operate on the quantum mechanical band-to-band tunneling (B2BT) principle [15-17]. A most claiming obstacle for TFET being low on-drive-current $\left(I_{o n}\right)$ because of less transmission probability $T_{W K B}$ of the interband tunneling barrier [18]. $T_{W K B}$ is estimated using WentzelKramer-Brillouin(WKB) approximation. WKB approximation defines it as exponential function of effective mass $m^{*}$, energy band gap $E_{g}$, tunneling length $\lambda$, and the difference in energy between both the source's conduction band and the channel's valence band $\delta \phi$. That is tunneling barrier approximation is estimated by $T_{W K B}$ $\approx \exp \left[\frac{-4 \lambda \cdot \sqrt{2 m^{*}} \cdot \sqrt{E_{g}^{3}}}{3 q \cdot \hbar\left(E_{g}+\delta \phi\right)}\right] . I_{o n}$ is obtained by integration of $T_{W K B}$ over depth of source-channel junction. Moreover, at fixed drain voltage, a rise in gate voltage $V_{g}$ changes the surface potential, minimizing $\lambda$ and raising $\delta \phi$. Thereby, maximum transmission probability $T_{W K B}$ 
of tunneling barrier implies a greater on-current $I_{o n}$. Accordingly, there are many possible causes to increase $I_{o n}$ : (i) The usage of high-K gate material, for example, is a type of gate dielectric engineering technology, service of multi-gate structures for controlling channel potential perfectly, selection of the optimum value of spacer to enhance on-current $I_{o n}$, asymmetric gate design by the use of different work functions and different gate dielectric materials, and ferroelectric gate oxide [19] (ii)Tunneling junction engineerings such as by use of the optimum value of source doping, by increasing effective tunneling area and heterojunction structure by use of low-bandgap materials [11] (iii) Material engineering techniques by use of unique materials like Ge and III-V semiconductors [20] (iv) by use of strainedsilicon [21]. However, the ferroelectric gate oxide technique is the best example of negative capacitance that can provide high $I_{\text {on }}$ and sub-60 $\mathrm{mV} /$ decade at a modest voltage value [22]. Integration of FE layer on TFET has drawn much attention in boosting the performance, i.e., possible structures for negative capacitance TFETs are metal- ferroelectric- insulator- semiconductor(MFIS), metal- ferro-electric -metal- insulator- semiconductor (MFMIS), metal- ferro-electric- semiconductor (MFS) and metal- ferroelectric- insulator- semiconductor- insulator- ferroelectric- metal (MFISIFM) [23]. It is well known that a combination of $\mathrm{FE}$ layers in the gate will provide internal voltage gain, which ultimately enhances tunneling probability $T_{W K B}$ and improves oncurrent [24]. Thus on-current is obtained by integrating tunneling probability $T_{W K B}$ over the source-channel junction. The proposed negative capacitance structure is MFISIFM, i.e., Ultra-thin body double gate FE layer TFET(UTB-DG-FE-TFET). The paper's organization follows with theory in part 2, followed by device construction and simulation setup in part 3 , following with results and discussion in part 4 . Lastly, part 5 concludes the paper.

\section{Theory}

The main reason for using the FE layer of $\mathrm{Si}: \mathrm{HFO}_{2}$ in UTB-DG-FE-TFET is to rectify the lattice mismatch problem. Thus, It is the most compatible with the flow of the CMOS manufacturing process [25]. The enticing characteristic of FE material is negative capacitance, which acts as a voltage step-up transformer. Therefore, SS and drain current are improved. Any CMOS device's subthreshold swing is generally calculated as the ratio of variation of gate bias potential $(\mathrm{Vg})$ to variation of subthreshold drain current by unity decade. Mathematically it can be expressed as:

$$
S S=\frac{\partial V_{g}}{\partial\left(\log _{10} I_{d}\right)}=\underbrace{\frac{\partial V_{g}}{\partial \psi_{s}}}_{\mathbf{m}} \cdot \underbrace{\frac{\partial \psi_{s}}{\partial\left(\log _{10} I_{d}\right)}}_{\mathbf{n}}
$$

Where $\mathrm{m}$ is the body factor used to calculate the effective twisting of surface potential at a specific bias, and $\mathrm{n}$ is the transport factor that tells how much current flows along the channel by lowering the potential. From Fig. 1, it is evident that the capacitor voltage divider rule clearly shows that $V_{g}$ and $\psi_{s}$ are correlated. that is

$m=\frac{\partial V_{g}}{\partial \psi_{s}}=1+\frac{C_{s}}{C_{e q}}$

where $\frac{1}{C_{e q}}=\frac{1}{C_{o x}}+\frac{1}{C_{f e}}$ is equivalent gate inter-facial capacitance, $C_{o x}$ is inter-facial layer capacitance, $C_{s}$ is semiconductor or intrinsic channel capacitance and $C_{f e}$ is ferroelectric layer negative capacitance. If $\mathrm{Q}$ is total charge observed by total capacitance across gate $C_{g}$ then equivalent gate inter-facial capacitance $C_{e q}$ is calculated by following expression: $C_{e q}=\frac{C_{o x} \cdot C_{f e}}{C_{o x}+C_{f e}}=$ $\frac{Q}{V_{g}-\psi_{s}}$. Effective voltage $\left(V_{g}-\psi_{s}\right)$ is seen across $C_{e q}$ and voltage in proportional with total charge $\mathrm{Q}$ also observed by $C_{g}$ being expressed by ( $\beta$.Q) where $\beta$ is internal amplification factor having value greater than unity. Therefore,

$$
\begin{aligned}
& \mathrm{Q}=C_{g}\left[\left(V_{g}-\psi_{s}\right)+\beta . \mathrm{Q}\right] \\
& \Rightarrow \mathrm{Q}=C_{g}\left(V_{g}-\psi_{s}\right)+C_{g} \beta . \mathrm{Q} \\
& \Rightarrow \mathrm{Q}\left(1-\beta . C_{g}\right)=C_{g}\left(V_{g}-\psi_{s}\right) \\
& \Rightarrow C_{e q}=\frac{Q}{\left(V_{g}-\psi_{s}\right)}=\frac{C_{g}}{\left(1-\beta \cdot C_{g}\right)}=\frac{C_{g}}{\left(1-V_{f b}\right)}
\end{aligned}
$$

If $V_{f b}=\beta . C_{g} \gg 1$ then equivalent gate- inter-facial capacitance $C_{e q}$ attain negative value; hence it is called negative capacitance TFET. In other sense, the feedback voltage $\left(V_{f b} \gg 1\right)$ must be positive to behave as negative capacitance TFET. It also implies amplifying internal voltage and reducing body factor $\mathrm{m}$ as clear from equation (2). Overall, SS is significantly decreased, as evident from equation (1). Moreover, equation (1) suggests that $\psi_{s} \gg V_{g}$, i.e., acts as a stepvoltage transformer which ultimately results in an improved electric field and enhanced on-current. In case of MOSFET, body factor $\mathrm{m}=1$ results into a lower limit of $\mathrm{SS}=60 \mathrm{mV} /$ decade i.e., equation (1) results into $\mathrm{SS}$ $\equiv \frac{\partial \psi_{s}}{\partial\left(\log _{10} I_{d}\right)}=\frac{K T}{q} \cdot \ln 10 \approx 60 \mathrm{mV} /$ decade. Moreover, ferroelectric polarization results in getting body factor $\mathrm{m}$ $<1$ in the proposed device structure. The benefits of using FE material on the top and bottom surface of the intrinsic channel over perovskite material like PZT and SBT is the best scaling in the nanometer range. Voltage across the MOS $\left(V_{M O S}\right)$ and voltage across the FE material $\left(V_{F E}\right)$ is related by $V_{g}=V_{M O S}+V_{F E}$. Voltage amplification is given by [26] : 
$A_{v}=\frac{V_{M O S}}{V_{g}}=\frac{\left|C_{f e}\right|}{\left|C_{f e}\right|-\left|C_{M O S}\right|}$. With $\left|C_{f e}\right|$ is less than $\left|C_{M O S}\right|$, voltage amplification factor yields into negative voltage gain.

\section{Device construction and simulation set up}

The cross-section perspective of the UTB-DG-FE-TFET is shown in Fig. 1, including its capacitive modeling. The channel is enclosed by an interfacial $\operatorname{layer}\left(\mathrm{SiO}_{2}\right)$ to reduce inter-diffusion. In a stacking having dielectric $\left(\mathrm{SiO}_{2}\right)$ on sidewalls, a ferroelectric sheet of silicon doped hafnium oxide $\left(\mathrm{Si}: \mathrm{HFO}_{2}\right)$ is utilized.

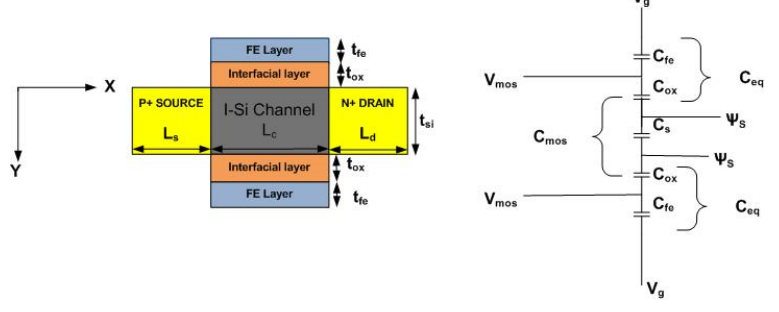

Fig. 1: UTB-DG-TFET Device Structure

Because of the lower value of the dielectric constant (32.5), Si: $\mathrm{HFO}_{2}$ is preferred over Strontium Bismuth Tantalate (SBT) and Lead Zirconate Titanate (PZT) to lessen the impacts of fringing $[27,28]$. It enables thinner ferroelectric layers. As a result, the gate stack ratio is better for scaling. Furthermore, $\mathrm{Si}: \mathrm{HFO}_{2}$ is compatible with the CMOS manufacturing process flow [17]. Design must always utilize an interfacial layer with the substrate to minimize lattice mismatch and enhance SS. However, the memory window is restricted owing to a voltage drop throughout the interfacial layer [29]. As a result, the best value of $t_{o x}=0.5 \mathrm{~nm}$ is chosen. The source is highly doped, via an impurity concentration of $10^{20} \mathrm{~cm}^{-3}$, compared to a weakly doped drain with an impurity concentration of $5 \times 10^{18} \mathrm{~cm}^{-3}$, to diminish ambipolar conduction. The channel is weakly doped, while not being entirely intrinsic, with a trivalent impurity concentration of $10^{16} \mathrm{~cm}^{-3}$. Table 1 lists the device variables used in the simulation. On the $2 \mathrm{D}$ TCAD Sentaurus Simulator, the device construction is simulated and tested [30]. Applying Fermi-Dirac statistics and the impact of band-gap narrowing, heavy doping at the drain, and source concerning channel is studied. Concentration-dependent mobility model, electric field-dependent mobility, band-gap narrowing model, Schockley-Read-Hall(SRH) recombination model, auger recombination model, and non-local band to band tunneling(BTBT) model are some of the models that are
Table 1: Experimental device parameters for the simulation

\begin{tabular}{|l|l|l|l|l|l|}
\hline Parameter & Symbol & Value & Parameter & Symbol & value \\
\hline $\begin{array}{l}\text { Gate } \\
\text { length }\end{array}$ & $L_{g}$ & $\begin{array}{l}15 \\
\mathrm{~nm}\end{array}$ & $\begin{array}{l}\text { Gate } \\
\text { Thick- } \\
\text { ness }\end{array}$ & $t_{g}$ & $\begin{array}{l}40 \\
\mathrm{~nm}\end{array}$ \\
\hline $\begin{array}{l}\text { Source } \\
\text { length }\end{array}$ & $L_{s}$ & $\begin{array}{l}20 \\
\mathrm{~nm}\end{array}$ & $\begin{array}{l}\text { Source } \\
\text { Thick- } \\
\text { ness }\end{array}$ & $t_{s}$ & $\begin{array}{l}30 \\
\mathrm{~nm}\end{array}$ \\
\hline $\begin{array}{l}\text { Drain } \\
\text { length }\end{array}$ & $L_{d}$ & $\begin{array}{l}20 \\
\mathrm{~nm}\end{array}$ & $\begin{array}{l}\text { Drain } \\
\text { Thick- } \\
\text { ness }\end{array}$ & $t_{d}$ & $\begin{array}{l}30 \\
\mathrm{~nm}\end{array}$ \\
\hline $\begin{array}{l}\text { Channel } \\
\text { length }\end{array}$ & $L_{c}$ & $\begin{array}{l}30 \\
\mathrm{~nm}\end{array}$ & $\begin{array}{l}\text { Interfacial } \\
\text { layer } \\
\text { thickness }\end{array}$ & $t_{o x}$ & $\begin{array}{l}0.5 \\
\mathrm{~nm}\end{array}$ \\
\hline $\begin{array}{l}\text { Ferroelectrid } \\
\text { layer } \\
\text { thickness }\end{array}$ & $t_{f e}$ & $2 \mathrm{~nm}$ & $\begin{array}{l}\text { Intrinsic } \\
\text { channel } \\
\text { thickness }\end{array}$ & $t_{s i}$ & $\begin{array}{l}10 \\
\mathrm{~nm}\end{array}$ \\
\hline
\end{tabular}

employed throughout computations. Ideal ferroelectric sheet having remanent polarisation $P_{r}$, coercive field $E_{c}$, and saturation polarisation $P_{s}$ are $10.75 \mu \mathrm{C} / \mathrm{cm}^{2}$, $1.15 \mathrm{MV} / \mathrm{Cm}$, and $11.37 \mu \mathrm{C} / \mathrm{cm}^{2}$, respectively, to eliminate hysteresis loss difficulties such as dc breakdown, retention, as well as fatigue [31]. For gate material, the proper work function $\left(\psi_{m}=4.6 \mathrm{eV}\right)$ is used.

\section{Simulation results and discussion}

The effect of ferroelectric thickness on electric parameters in UTB-DG-FE-TFET is discussed. The paper investigates a comparison of UTB-DG-FE-TFET with FE-TFET [32] and conventional TFET [33,34]. Simulated data of UTB-DG-FE-TFET is compared with reference data of FE-TFET and conventional TFET, which shows proposed UTB-DG-FE-TFET, which indicates the high value of on current $I_{o n}$ and significant change in off current $I_{o f f}$. In addition, it must be noted that due to the negative capacitance but the double gate construction, the drain current level rises. The drain current $I_{d s}$ rises as the gate voltage $V_{g s}$ increases. Memory window is key deciding parameter for description of total dipoles and can be expressed as $\mathrm{MW} \equiv$ $2 E_{c} \times t_{f e}$, here $E_{c}$ as well as $t_{f e}$ denote coercive field or coercivity and ferroelectric material thickness, respectively [25]. In Table 2, the proposed work's electrical parameters have been compared to those of previously reported devices.

\subsection{Comparison of TCAD model physics with referenced data}

Transfer characteristics of UTB-DG-FE-TFET are examined and simulated using experimental parameters shown in Table. 1 and also compared with FE-TFET 
[32] and conventional TFET [33], respectively in Fig. 2. Proposed UTB-DG-FE-TFET almost provides two times improvement in $I_{o n}$ and 20 times improvement in $I_{o n}$ in comparison with FE-TFET and conventional TFET, respectively. One can observe that the proposed structure also gives $10^{1}$ times reduction in $I_{\text {off }}$ and $10^{2}$ times reduction in $I_{\text {off }}$ as compared to FE-TFET and conventional TFET, respectively. Internal voltage amplification, such as that provided by a voltage step-up transformer, is the cause of this occurrence, which finally yields into increment in $I_{o n}$ and reduction in $I_{o f f}$, i.e., it leads to enhancement in the current level.

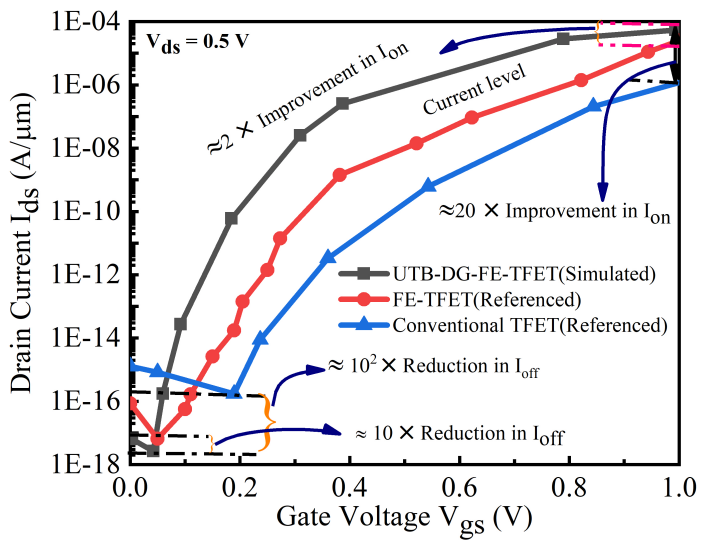

Fig. 2: Simulated and referenced data comparison [Under forward sweep at $V_{d s}=0.5 \mathrm{~V}$ for quantitative comparison]

Due to the particular hysteresis behavior of FE materials, forward and reverse sweeps for voltage are introduced in transfer characteristics for the proposed structure in Fig. 3.

Comparison of output characteristics of UTB-DGFE-TFET with FE-TFET and conventional TFET at $V_{g s}=1 \mathrm{~V}$ is illustrated in Fig. 4. In triode region up to $V_{d s}=0.2 \mathrm{~V}$, a channel is formed due to the availability of large charge carriers, and when the $V_{d s}$ is increased beyond $0.2 \mathrm{~V}$, the large numbers of charge carries starts to tunnel. Thus, $I_{d s}$ shows a growing exponential curve due to the lowering of drain induced barrier. The lateral electrical field from the drain is halted through the channel. That's why a perfect saturation is also seen in output characteristics [35].

Moreover, incomplete charge compensation and decrease in polarization also happened due to depolarization field in FE materials. Fig. 5 illustrates that approximately the same window is granted for two different structures of $15 \mathrm{~nm}$ gate length UTB-DG-FETFET and $30 \mathrm{~nm}$ gate length FE-TFET by sweeping

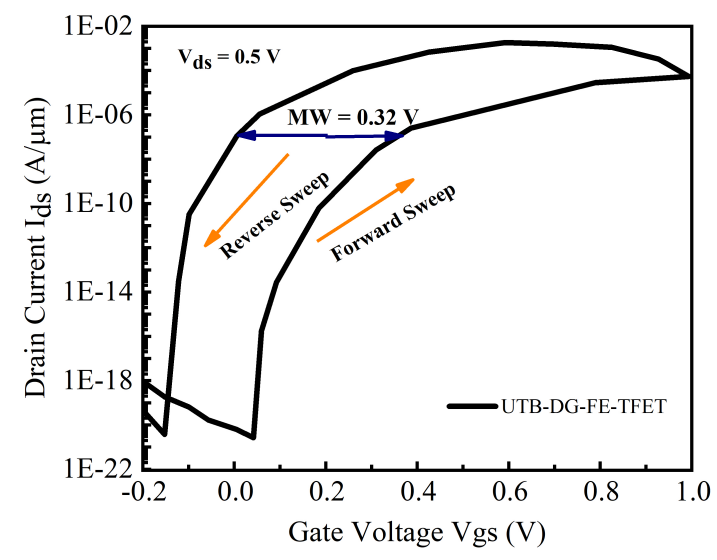

Fig. 3: Transfer characteristics of UTB-DG-FE-TFET under forward and reverse sweep at $V_{d s}=0.5 \mathrm{~V}$ keeping all parameters same

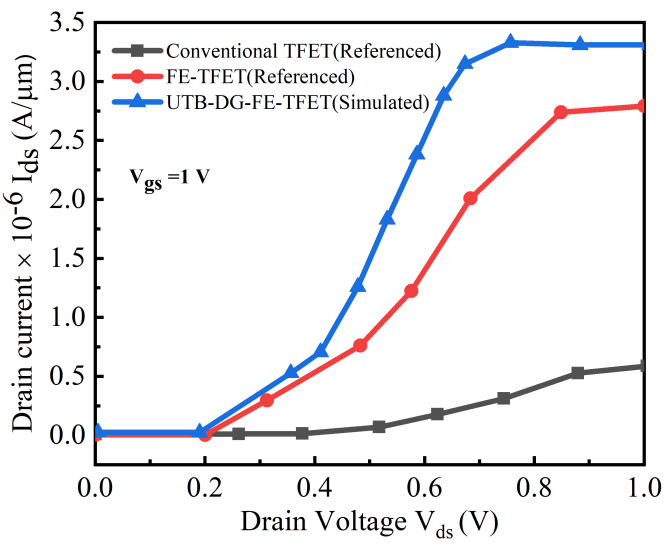

Fig. 4: Comparison of output characteristics of UTB-DGFE-TFET with FE-TFET and conventional TFET at $V_{g s}=$ $1 \mathrm{~V}$ keeping all parameters same

the gate voltage. Comparison results confirm that approximately the same memory window $(0.32 \mathrm{~V}$ for 15 $\mathrm{nm}$ gate length UTB-DG-FE-TFET and $0.32 \mathrm{~V}$ for 40nm gate length FE-TFET) is obtained. Moreover, One can observe an enhancement in $I_{o n}$ and also a reduction in $I_{o f f}$. Thereby $15 \mathrm{~nm}$ gate length UTB-DGFE-TFET structure could be the best alternative for enriched electric performance. Apart from data storage properties of FE materials, the surface potential is boosted, and the output current level is amplified [24]. For this purpose, the variation in the channel surface potential of UTB-DG-FE-TFET, FE-TFET, and conventional TFET to the varied value of $V_{g s}$ is presented in Fig. 6. Fig. 7 demonstrates the comparison of UTBDG-FE-TFET electrical fields with FE-TFET and conventional TFET [27]. Under the bias conditions having 


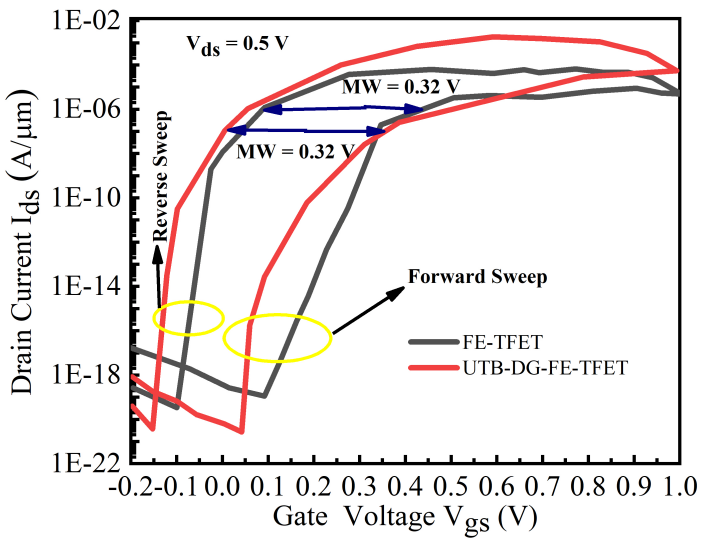

Fig. 5: Comparative analysis of transfer characteristics for UTB-DG-FE-TFETs with $15 \mathrm{~nm}$ gate length and FE-TFETs with $30 \mathrm{~nm}$ gate length while keeping all other parameters constant

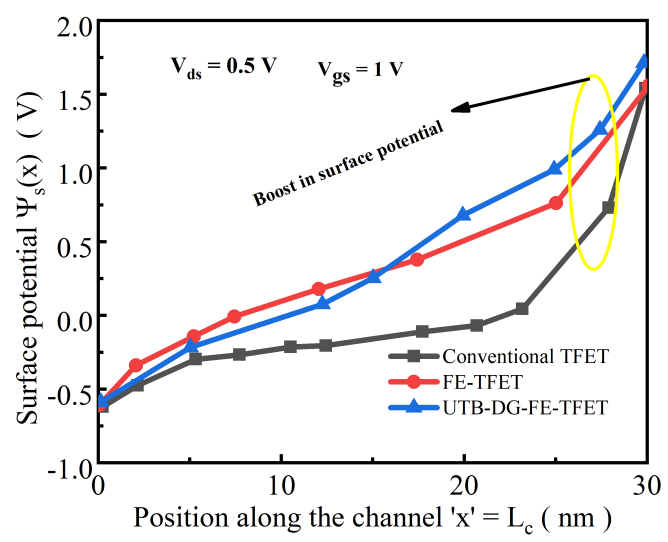

Fig. 6: Surface potential $\psi(\mathrm{x})$ variation across the channel positions of UTB-DG-FE-TFET, FE-TFET, and conventional TFET with channel length $L c=30 \mathrm{~nm}$ at constant $V d s=0.5 \mathrm{~V}$ and $V g s=1 \mathrm{~V}$. TCAD simulated data with the same variables as stated in Table 1 is depicted by symbols.

$V_{g s}=1 \mathrm{~V}$ and $V_{d s}=0.5 \mathrm{~V}$, the maximum electric field for UTB-DG-FE-TFET at the interface of source and channel is $37.5 \%$ greater than conventional TFET and $10 \%$ greater than FE-TFET. This is owing to the excellent characteristics of positive feedback caused by FE materials.

4.2 Effect of ferrothickness on subthreshold swing, threshold voltage, electric field dependent polarization and Ion/Ioff

Due to negative capacitance, voltage is amplified to a great extent but with negative potential gain. Sub-

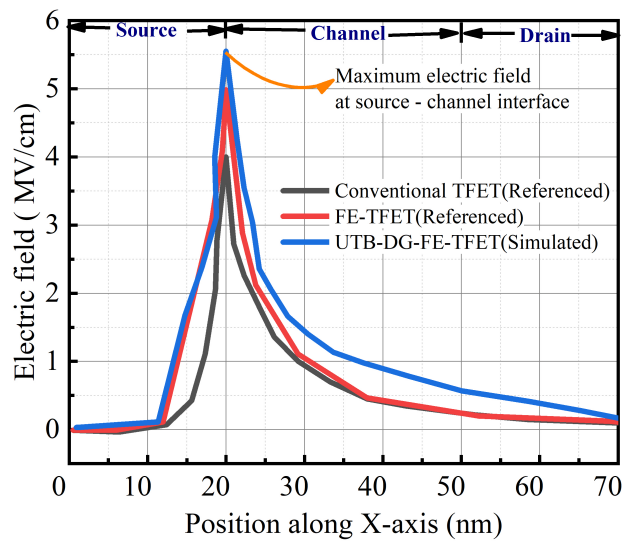

Fig. 7: Comparison of electric fields for UTB-DG-FE-TFET with FE-TFET and conventional TFET

threshold swing is a crucial parameter for understanding any MOS switching behavior [4]. It is found that Polarization of ferroelectric layer improves the value of point subthreshold swing $S S_{\text {point }}$ as shown in Fig. 8. Fig. 8. shows that as $t_{f e}$ varies from 4 to $16 \mathrm{~nm}, S S_{\text {point }}$ value is reported below $36 \mathrm{mV} /$ decade for a range of approximately ten orders of magnitude. Average subthreshold swing $S S_{\text {average }}$ is noticed around below 45 $\mathrm{mV} /$ decade in Fig. 9 for the various value of $t_{f e}$. Because the subthreshold properties are significantly improved, the device is appropriate for ultra-low energy switching applications.

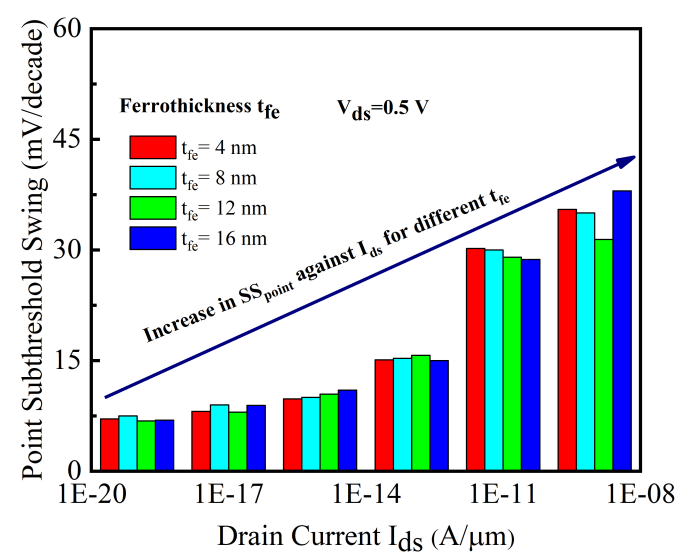

Fig. 8: Impact of ferrothickness $t_{f e}$ on $S S_{\text {point }}$

Fig. 10 demonstrates the relation between threshold voltage $V_{t h}$ and intrinsic channel thickness $t_{s i}$ for different values of $t_{f e}$. The figure reveals that as the intrinsic channel thickness $t s i$ and the FE layer thickness 


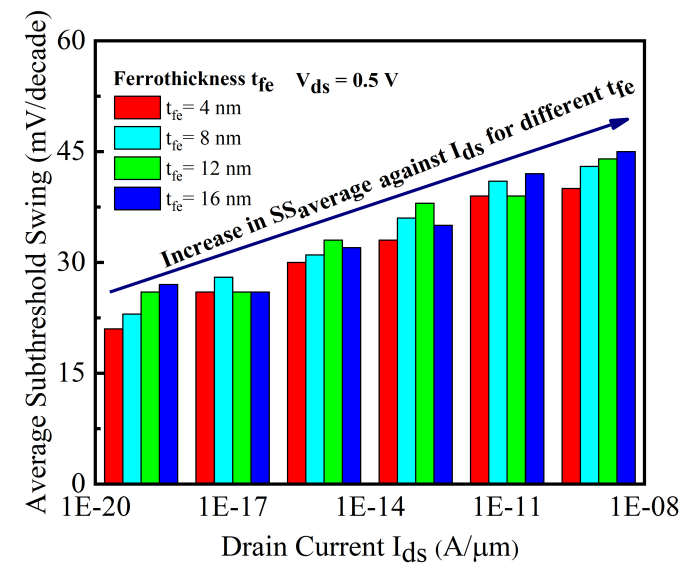

Fig. 9: Impact of ferrothickness $t_{f e}$ on $S S_{\text {average }}$

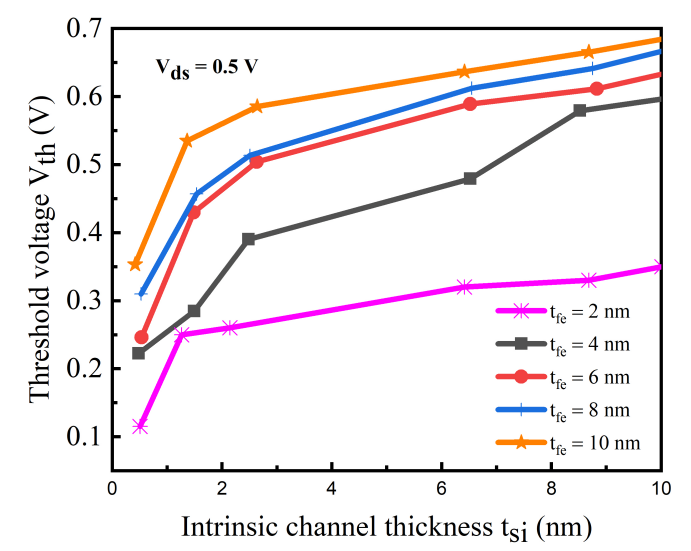

Fig. 10: Threshold voltage $V_{t h}$ against intrinsic channel thickness $t_{s i}$ for different FE layer thickness $t_{f e}$

$t_{f e}$ increases, so does $V_{t h}$. This was primarily because the tunneling volume was reduced with a rise in $t_{s i}$ and the feedback charge lowered since $t_{f e}$ increased $[27,33]$. A FE layer of $t_{f e}=16 \mathrm{~nm}$ shows a relatively thinner hysteresis curve having greater polarization on the application of the highest electric field to get deep saturation that is evident from Fig. 11. Therefore current level enhances in thinner FE layer with maximum negative capacitance. Due to spontaneous polarization in ferroelectric, a non-linear relationship exists between polarization and electric field, represented by a hysteresis loop. On increasing $t_{f e}$, magnetic polarization tries to become zero, as illustrated in Fig.11. Thereby Coercive field strength reduces in-considerably. Therefore, simulation results validate that characteristics governing parameter are $t_{f e}$ and memory window value is raised dramatically. The primary concern in the design of the proposed device is to choose an optimum value of FE layer thickness so that three key parameters SS, memory window, and drain current, are improved. Therefore former is reduced, and the latter is increased. Recently explored silicon doped hafnium oxide ( $\mathrm{Si}: \mathrm{HFO}_{2}$ ) is selected not only to provide relatively coercive field $E_{c}$ over PZT (Lead-Zirconate-Titanate) and SBT (Strontium-Barium-Titanate) but also to present a large memory window [25]. Fig. 12 depicts a summary

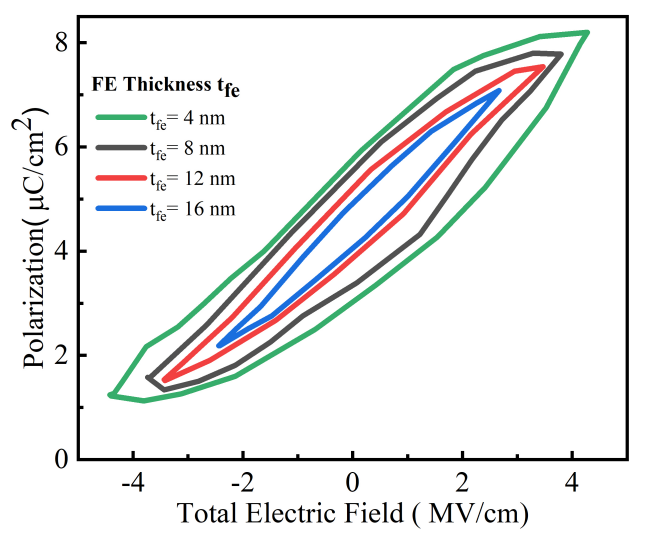

Fig. 11: Total electric field vs. Polarization for different FE layer thickness

of the impact of the ferroelectric device layer thickness on the $I_{o n} / I_{\text {off }}$ ratio of the ferroelectric device. Fig. 12 illustrates that the ON current rises as the thickness of the ferroelectric layer decreases due to polarisation trapped charges while the OFF current remains similar. This improves the ratio of Ion/Ioff, as the ferroelectric layer's thickness decreases.

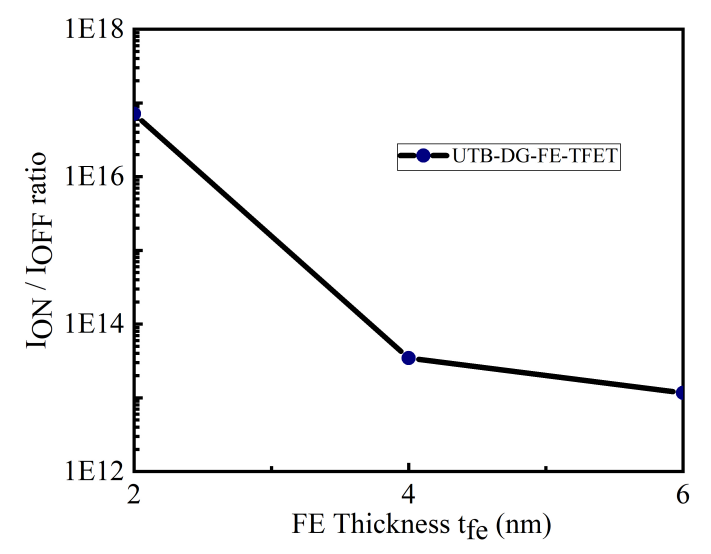

Fig. 12: Change in $I_{o n} / I_{o f f}$ ratio with ferrothickness $t_{f e}$ 
Table 2: Comparison of electrical characteristics with previously published works $[25,26,28,29]$

\begin{tabular}{|c|c|c|c|c|}
\hline Variation & $\begin{array}{c}\text { Ferro } \\
\text { thickness } \\
(\mathrm{nm})\end{array}$ & $\begin{array}{c}I_{\text {on }} / I_{\text {off }} \\
\text { ratio }\end{array}$ & $\begin{array}{c}\text { MW } \\
(\mathrm{V})\end{array}$ & $\begin{array}{c}S S_{\text {point }} \\
(\mathrm{mV} / \mathrm{dec})\end{array}$ \\
\hline \multirow{2}{*}{$\begin{array}{c}\text { Proposed } \\
\text { Work }\end{array}$} & 2 & $7.117 \times 10^{13}$ & 0.32 & 23 \\
\cline { 2 - 5 } & 4 & $3.459 \times 10^{11}$ & 0.25 & 19.6 \\
\hline \multirow{2}{*}[28]{} & 6 & $1.165 \times 10^{11}$ & 0.21 & 17.3 \\
\cline { 2 - 5 } & 30 & $5 \times 10^{13}$ & 1.8 & - \\
\hline \multirow{3}{*}[25]{} & 10 & $1 \times 10^{12}$ & 4 & - \\
\cline { 2 - 5 } & 15 & $9 \times 10^{6}$ & 1.7 & - \\
\cline { 2 - 5 } & 20 & $2 \times 10^{6}$ & 2.05 & - \\
\hline$[29]$ & 100 & $1 \times 10^{7}$ & 1.8 & - \\
\hline$[26]$ & 153 & $1 \times 10^{10}$ & - & 5 \\
\hline
\end{tabular}

At $t_{f e}=2 \mathrm{~nm}$, it was discovered that the proposed device had a better SS, a large ratio of $I_{o n} / I_{o f f}$, and a larger memory window. Since the ferroelectric layer is placed on top and bottom of the gate dielectric. The ferroelectric layer thickness is controlled to get the best band to band tunneling (BTBT).

4.3 Effect of device geometry variants on transfer characteristics

Fig. 13 illustrates the logarithmic effect of ferroelectric layer thickness on drain current while changing gate voltage on a linear scale. Table 3 shows the ferroelectric characteristics for different $\mathrm{Si}: \mathrm{HFO}_{2}$ layer thicknesses. Table 4 displays the device's parameter value concerning different device geometry variations. Table 4 demonstrates that improved SS, large memory window and high on-off current ratio are regarded to be the most suitable value when compared with other variations at $t_{f e}=2 \mathrm{~nm}, t_{o x}=0.5 \mathrm{~nm}, t_{s i}=10 \mathrm{~nm}, S_{i} O_{2}$ as interfacial layer type and $L_{c}=30 \mathrm{~nm}$.

The ferroelectric layer thickness has a significant effect on device performance. Negative capacitance rises as $t_{f e}$ decreases resulting in increased trapped charge density. These trapped charge densities within this ferroelectric sheet create a channel and start a tunneling process at low $\mathrm{Vgs}$. It can be shown that when the thickness of ferroelectric oxide $t_{f e}$ increases from $2 \mathrm{~nm}$ to $4 \mathrm{~nm}, 6 \mathrm{~nm}$, etc., the drain current significantly reduces. With an increase in $t_{f e}$, this results in the creation of a low feedback charge. However, in the absence of the FE layer $\left(t_{f e}=0\right)$, the drain current is observed to be less because of the absence of positive feedback. For $t_{f e}>0$, a high current is seen due to positive feedback introduced by negative capacitance. Yet the coercive field $E_{c}$ hardly reduces on increasing $t_{f e}$. So it leads to an apparent dependency of $t_{f e}$ on the memory window

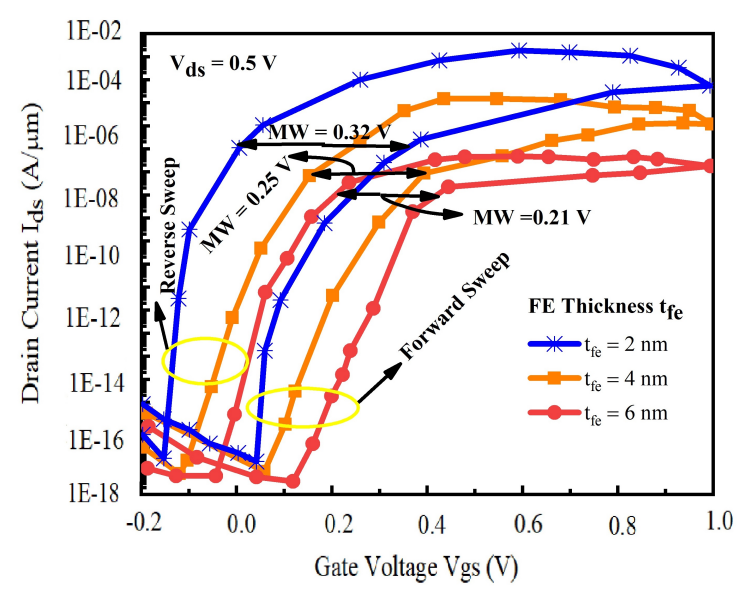

Fig. 13: Impact of different FE layer thickness on transfer characteristics

Table 3: Si: $\mathrm{HFO}_{2}$ ferroelectric characteristics increasing $t_{f e}$

\begin{tabular}{|c|c|c|c|c|}
\hline $\begin{array}{c}t_{f e} \\
(\mathrm{~nm})\end{array}$ & $\epsilon_{r}$ & $\begin{array}{c}P_{r} \\
\left(\mu \mathrm{C} / \mathrm{cm}^{2}\right)\end{array}$ & $\begin{array}{c}P_{s} \\
\left(\mu \mathrm{C} / \mathrm{cm}^{2}\right)\end{array}$ & $\begin{array}{c}E_{c} \\
(\mathrm{MV} / \mathrm{cm})\end{array}$ \\
\hline 2 & 37.2 & 13.5 & 13.7 & 1.52 \\
\hline 4 & 35.8 & 12.1 & 12.2 & 1.48 \\
\hline 6 & 33.1 & 10.65 & 10.7 & 1.44 \\
\hline
\end{tabular}

(MW). For this reason, MW rises from $0.32 \mathrm{~V}$ to 0.45 V substantially.

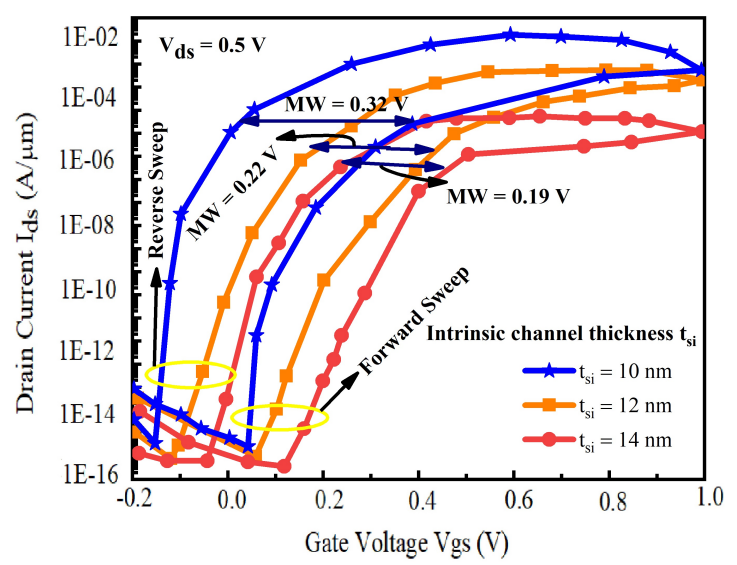

Fig. 14: Influence of various intrinsic channel thickness on transfer characteristics

Fig. 14 illustrates the influence of the thickness of the channel of varying the intrinsic channel thickness $t_{s i}$ on the $I_{d s^{-}} V_{g s}$ curve. The drain current $I_{d s}$ decreases with $t_{s i}$, as shown in the figure. The reason for this is that when $t_{s i}$ rises, the tunneling volume decreases. Moreover, one can notice that the memory window (MW) falls with $t_{s i}$ due to a reduction in the 
Table 4: EXTRACTED PARAMETER VALUE

\begin{tabular}{|l|l|l|l|l|l|}
\hline $\begin{array}{l}\text { Device } \\
\text { geometry } \\
\text { variants }\end{array}$ & $\begin{array}{l}\text { MW } \\
(\mathrm{V})\end{array}$ & $\begin{array}{l}V_{t h} \\
(\mathrm{~V})\end{array}$ & SS & $I_{\text {on }} / I_{\text {off }}$ \\
\hline \multirow{3}{*}{$t_{f e}(\mathrm{~nm})$} & 2 & 0.32 & 0.38 & 23 & $7.1 \times 10^{13}$ \\
\cline { 2 - 6 } & 4 & 0.25 & 0.59 & 19.6 & $3.4 \times 10^{11}$ \\
\cline { 2 - 6 } & 6 & 0.21 & 0.61 & 17.3 & $1.1 \times 10^{11}$ \\
\hline \multirow{4}{*}{$t_{s i}(\mathrm{~nm})$} & 10 & 0.32 & 0.38 & 23 & $4.1 \times 10^{12}$ \\
\cline { 2 - 6 } & 12 & 0.22 & 0.50 & 26.5 & $2.3 \times 10^{11}$ \\
\cline { 2 - 6 } & 14 & 0.19 & 0.51 & 21.3 & $1.2 \times 10^{10}$ \\
\hline $\begin{array}{l}\text { Interfacial } \\
\text { layer } \\
\text { types }\end{array}$ & $\mathrm{HFO}_{2}$ & 0.56 & 0.31 & 20.6 & $3.1 \times 10^{12}$ \\
\cline { 2 - 6 } & $\mathrm{Si}_{3} N_{4}$ & 0.41 & 0.32 & 21.5 & $5.1 \times 10^{11}$ \\
\cline { 2 - 6 }$t_{\text {ox }}(\mathrm{nm})$ & $\mathrm{SiO}_{2}$ & 0.32 & 0.38 & 23 & $2.4 \times 10^{10}$ \\
\cline { 2 - 6 } & 0.25 & 0.31 & 0.45 & 38.5 & $2.3 \times 10^{12}$ \\
\cline { 2 - 6 } & 0.5 & 0.41 & 0.42 & 31.3 & $4.6 \times 10^{12}$ \\
\hline \multirow{3}{*}{$\begin{array}{l}L_{c} \\
(\mathrm{~nm})\end{array}$} & 10 & 0.37 & 0.38 & 23 & $3.1 \times 10^{11}$ \\
\cline { 2 - 6 } & 20 & 0.41 & 0.36 & 13.7 & $5.6 \times 10^{11}$ \\
\cline { 2 - 6 } & 30 & 0.58 & 0.65 & 23.4 & $4.8 \times 10^{12}$ \\
\cline { 2 - 6 } & 70 & 0.58 & 0.75 & 37.6 & $6.8 \times 10^{11}$ \\
\hline
\end{tabular}

coercive field $E_{c}$. Fig. 15 shows the impact of various

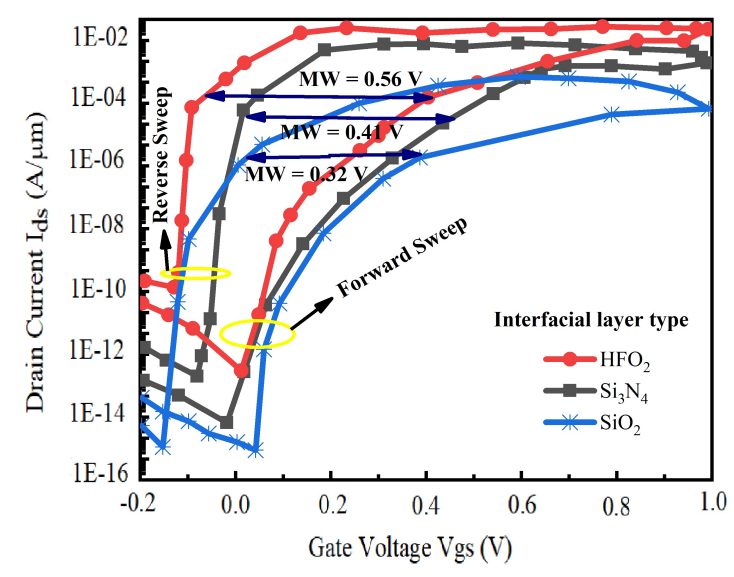

Fig. 15: The effect of various interfacial layer types on transfer characteristics

inter-facial layer types, i.e., $0.5 \mathrm{~nm} \mathrm{SiO}_{2}$ layer is replaced by $\mathrm{HFO}_{2}$ and then by $\mathrm{Si}_{3} \mathrm{~N}_{4}$. Detailed analysis is done by $\mathrm{SiO}_{2}$ only because of two limitations of $\mathrm{HFO}_{2}$ : (i) lattice mismatch problem between $\mathrm{HFO}_{2}$ and silicon substrate during fabrication process (ii) deterioration of $I_{o n} / I_{o f f}$, instead of large ON current and large memory window of $0.56 \mathrm{~V}$. Impact of various interfacial layer thickness $t_{o x}$ of $\mathrm{SiO}_{2}$ interfacial layer on transfer curve $I_{d s^{-}} V_{g s}$ is depicted in Fig. 16. For the moderate value of $t_{o x}=0.5 \mathrm{~nm}$, the enormous electric field is induced by a more significant voltage drop in the substrate material at the tunneling interface. Thus on current increases with greater memory window as compared to a thicker layer of $t_{o x}=1 \mathrm{~nm}$. Effective oxide thickness $t_{o x}=0.5 \mathrm{~nm}$ is one of optimum dimension

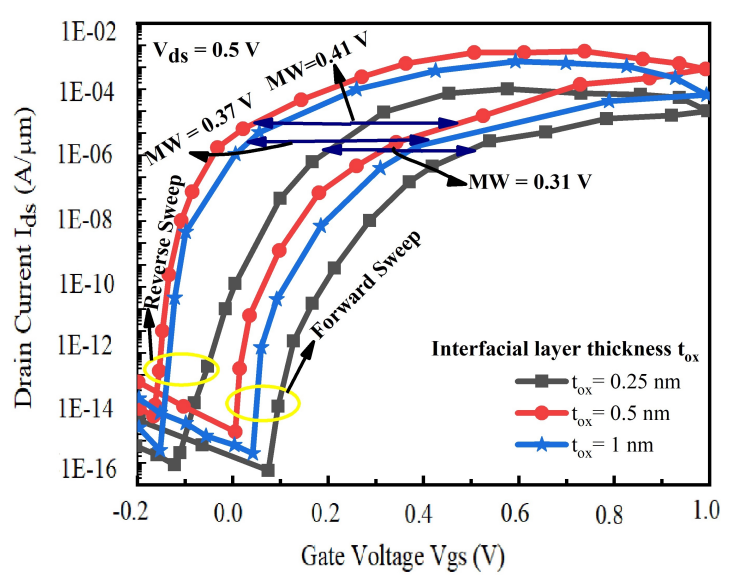

Fig. 16: The effect of various interfacial layer thicknesses upon transfer curve

over standard CMOS high dielectric/metal gate technique. In addition, for $t_{o x}=0.25 \mathrm{~nm}$, on current is less with reduced memory window. Effect of different chan-

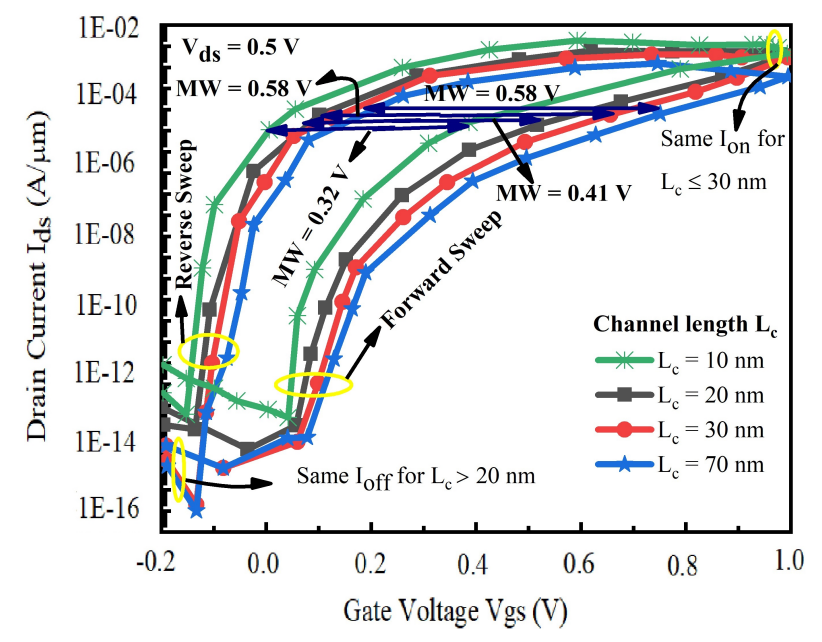

Fig. 17: Impact of different channel length on transfer characteristics

nel length $L_{c}$ on $I_{d s}-V_{g s}$ transfer curve is depicted in Fig. 17. It is noticed from the figure that $I_{\text {on }}$ remains the same for channel length up to $30 \mathrm{~nm}$ while the same $I_{o f f}$ is obtained for channel length greater than $20 \mathrm{~nm}$. The figure shows that on-current $I_{o n}$ decreases for channel length greater than $30 \mathrm{~nm}$, and off-current $I_{\text {off }}$ rises for channel length smaller than $20 \mathrm{~nm}$. The best suitable channel length $L_{c}=30 \mathrm{~nm}$ is chosen because of the larger $I_{o n}$ and memory window. 


\section{Conclusions}

UTB-DG-FE-TFET is experimentally demonstrated to investigate electrical characteristics like surface potential, drain current, threshold voltage, and subthreshold swing over conventional TFET and FE-TFET using Sentaurus TCAD 2D simulator. The results indicate that the current level is raised by increasing the gate voltage, with a drop in the off-current compared to other TFETs. It is also noticed that surface potential is boosted along with the channel position, and at the source-channel interface, there seems to be a maximum electrical field. In contrast, $V_{t h}$ rises with increment in $t_{s i}$ and $t_{f e}$ and thinnest hysteresis loop is obtained for larger $t_{f e}$. It is also noticed that $S S_{\text {point }}$ and $S S_{\text {average }}$ are improved against $I_{d s}$ for different value of $t_{f e}$. Moreover, Effect of larger $t_{f e}, t_{s i}$ results into reduced tunneling current. While the type of interfacial layer, especially $\mathrm{SiO}_{2}$ layer having $t_{o x}=0.5 \mathrm{~nm}$ over $\mathrm{HFO}_{2}$ and $S i_{3} N_{4}$ is found to be best suitable because of lattice mismatch problem faced by $\mathrm{HFO}_{2}$ against silicon substrate. In addition, larger on-current as well as huge width of memory window is noted for $t_{o x}=0.5 \mathrm{~nm}$. Finally, channel length $L_{c}$ also has a significant effect on transfer characteristics. Furthermore, a high Ion and a very little $\operatorname{Iof} f$ was shown for the proposed design to produce outstanding Ion/Ioff $\left(\approx 10^{13}\right)$. Thus, Such TFETs may be appealing for ultra-low-power device operation while still providing high performance.

Acknowledgements The authors would like to acknowledge the support of the VLSI Laboratory at MNIT, Jaipur, originated from the SMDP-C2SD Project funded by MeitY, Govt. of India, for providing all the facilities for carrying out the work.

\section{Declarations}

\section{Funding statement}

The authors received no financial support for their research, writing, or publication of this paper.

\section{Conflicts of interest/Competing interests}

The authors declare that they have no conflict of interest.

\section{Availability of data and material}

Not applicable

\section{Code availability}

Not applicable

\section{Compliance with ethical standards}

In research involving human participants, all procedures were carried out in compliance with ethical guidelines.

\section{Consent to participate}

Not applicable

\section{Consent for Publication}

Not applicable

\section{Author contributions}

\section{Girdhar Gopal}

Conceptualization, TCAD Simulation, Writing and Editing.

\section{Tarun Varma}

Supervision, Reviewing, Investigation and Validation.

\section{References}

1. T. Nirschl, J. Fischer, M. Fulde, A. Bargagli-Stoffi, M. Sterkel, J. Sedlmeir, C. Weber, R. Heinrich, U. Schaper, J. Einfeld, et al., "Scaling properties of the tunneling field effect transistor (tfet): Device and circuit," Solid-state electronics, vol. 50, no. 1, pp. 44-51, 2006.

2. B. Jena, K. Bhol, U. Nanda, S. Tayal, and S. R. Routray, "Performance analysis of ferroelectric gaa mosfet with metal grain work function variability," Silicon, pp. 1-8, 2021.

3. K. Vanlalawmpuia and B. Bhowmick, "Linearity performance analysis due to lateral straggle variation in heterostacked tfet," Silicon, pp. 1-7, 2019.

4. F. I. Sakib, F. E. Mullick, S. Shahnewaz, S. Islam, and M. Hossain, "Influence of device architecture on the performance of negative capacitance mfmis transistors," Semiconductor Science and Technology, vol. 35, no. 2, p. $025005,2019$.

5. S. Salahuddin and S. Datta, "Can the subthreshold swing in a classical fet be lowered below $60 \mathrm{mv} /$ decade?," in 2008 IEEE International Electron Devices Meeting, pp. 1-4, IEEE, 2008. 
6. S. Tayal, J. Ajayan, L. L. Joseph, J. Tarunkumar, D. Nirmal, B. Jena, and A. Nandi, "A comprehensive investigation of vertically stacked silicon nanosheet field effect transistors: an analog/rf perspective," Silicon, pp. 1-8, 2021.

7. Q. Zhang, W. Zhao, and A. Seabaugh, "Lowsubthreshold-swing tunnel transistors," IEEE Electron Device Letters, vol. 27, no. 4, pp. 297-300, 2006.

8. S. Tayal, G. Vibhu, S. Meena, and R. Gupta, "Optimization of device dimensions of high-k gate dielectric based dg-tfet for improved analog/rf performance," Silicon, pp. 1-7, 2021.

9. T. Varma, C. Periasamy, and D. Boolchandani, "Performance evaluation of bottom gate zno based thin film transistors with different w/l ratios for uv sensing," $S u$ perlattices and Microstructures, vol. 114, pp. 284-295, 2018.

10. A. Ionescu and H. Riel, "Tunnel field-effect transistors as energy-efficient electronic switches," Nature, vol. 479, pp. 329-37, 112011.

11. E.-H. Toh, G. H. Wang, G. Samudra, and Y.-C. Yeo, "Device physics and design of germanium tunneling fieldeffect transistor with source and drain engineering for low power and high performance applications," Journal of Applied Physics, vol. 103, no. 10, p. 104504, 2008.

12. K. Gopalakrishnan, P. Griffin, and J. Plummer, "I-mos: a novel semiconductor device with a subthreshold slope lower than kt/q," in Digest. International Electron Devices Meeting,, pp. 289-292, 2002.

13. H. Kam, D. T. Lee, R. T. Howe, and T.-J. King, "A new nano-electro-mechanical field effect transistor (nemfet) design for low-power electronics," in IEEE InternationalElectron Devices Meeting, 2005. IEDM Technical Digest., pp. 463-466, IEEE, 2005.

14. A. Saeidi, F. Jazaeri, F. Bellando, I. Stolichnov, G. V. Luong, Q.-T. Zhao, S. Mantl, C. C. Enz, and A. M. Ionescu, "Negative capacitance as performance booster for tunnel fets and mosfets: an experimental study," IEEE electron device letters, vol. 38, no. 10, pp. 1485-1488, 2017.

15. C. $\mathrm{Hu}$, "Green transistor as a solution to the ic power crisis," in 2008 9th International Conference on Solid-State and Integrated-Circuit Technology, pp. 16-20, IEEE, 2008.

16. P. Ghosh and B. Bhowmick, "Effect of temperature on reliability issues of ferroelectric dopant segregated schottky barrier tunnel field effect transistor (fe ds-sbtfet)," Silicon, vol. 12, no. 5, pp. 1137-1144, 2020.

17. P. P. Goswami and B. Bhowmick, "Optimization of electrical parameters of pocket doped soi tfet with 1 shaped gate," Silicon, vol. 12, no. 3, pp. 693-700, 2020.

18. A. Saeidi, F. Jazaeri, I. Stolichnov, G. V. Luong, Q.-T. Zhao, S. Mantl, and A. M. Ionescu, "Effect of hysteretic and non-hysteretic negative capacitance on tunnel fets dc performance," Nanotechnology, vol. 29, no. 9, p. 095202 , 2018.

19. K. Bhuwalka, J. Schulze, and I. Eisele, "Scaling the vertical tunnel fet with tunnel bandgap modulation and gate workfunction engineering," IEEE Transactions on Electron Devices, vol. 52, no. 5, pp. 909-917, 2005.

20. S. Shekhar, J. Madan, and R. Chaujar, "Source/gate material-engineered double gate tfet for improved $\mathrm{rf}$ and linearity performance: a numerical simulation," Applied Physics A, vol. 124, no. 11, pp. 1-10, 2018.

21. G. V. Luong, S. Strangio, A. Tiedemann, P. Bernardy, S. Trellenkamp, P. Palestri, S. Mantl, and Q. Zhao, "Strained silicon complementary tfet sram: Experimental demonstration and simulations," IEEE journal of the Electron Devices Society, vol. 6, pp. 1033-1040, 2018.
22. S. Salahuddin and S. Datta, "Use of negative capacitance to provide voltage amplification for low power nanoscale devices," Nano letters, vol. 8, no. 2, pp. 405-410, 2008.

23. G. Pahwa, T. Dutta, A. Agarwal, and Y. S. Chauhan, "Compact model for ferroelectric negative capacitance transistor with mfis structure," IEEE Transactions on Electron Devices, vol. 64, no. 3, pp. 1366-1374, 2017.

24. M.-H. Lee, Y.-T. Wei, J.-C. Lin, C.-W. Chen, W.-H. Tu, and M. Tang, "Ferroelectric gate tunnel field-effect transistors with low-power steep turn-on," AIP Advances, vol. 4, no. 10, p. 107117, 2014.

25. A. Saeidi, A. Biswas, and A. M. Ionescu, "Modeling and simulation of low power ferroelectric non-volatile memory tunnel field effect transistors using silicon-doped hafnium oxide as gate dielectric," Solid-State Electronics, vol. 124, pp. 16-23, 2016

26. C. Liu, P.-G. Chen, M.-J. Xie, S.-N. Liu, J.-W. Lee, S.J. Huang, S. Liu, Y.-S. Chen, H.-Y. Lee, M.-H. Liao, et al., "Simulation-based study of negative-capacitance double-gate tunnel field-effect transistor with ferroelectric gate stack," Japanese Journal of Applied Physics, vol. 55, no. 4S, p. 04EB08, 2016.

27. S. Kumar, E. Goel, K. Singh, B. Singh, M. Kumar, and S. Jit, "A compact 2-d analytical model for electrical characteristics of double-gate tunnel field-effect transistors with a sio 2 /high- $k$ stacked gate-oxide structure," IEEE Transactions on Electron Devices, vol. 63, no. 8, pp. 3291-3299, 2016.

28. S. Mueller, E. Yurchuk, S. Slesazeck, T. Mikolajick, J. Müller, T. Herrmann, and A. Zaka, "Performance investigation and optimization of si: Hfo 2 fefets on a 28 nm bulk technology," in 2013 Joint IEEE International Symposium on Applications of Ferroelectric and Workshop on Piezoresponse Force Microscopy (ISAF/PFM), pp. 248-251, IEEE, 2013.

29. A. Rusu, G. A. Salvatore, D. Jiménez, and A. M. Ionescu, "Metal-ferroelectric-meta-oxide-semiconductor field effect transistor with sub-60mv/decade subthreshold swing and internal voltage amplification," in 2010 International Electron Devices Meeting, pp. 16.3.1-16.3.4, 2010.

30. T. Synopsys, "Sentaurus device user's manual," Mountain View, CA, 2009.

31. A. Biswas, N. Dagtekin, W. Grabinski, A. Bazigos, C. Le Royer, J.-M. Hartmann, C. Tabone, M. Vinet, and A. M. Ionescu, "Investigation of tunnel field-effect transistors as a capacitor-less memory cell," Applied Physics Letters, vol. 104, no. 9, p. 092108, 2014.

32. A. M. Ionescu, L. Lattanzio, G. A. Salvatore, L. De Michielis, K. Boucart, and D. Bouvet, "The hysteretic ferroelectric tunnel fet," IEEE transactions on electron devices, vol. 57, no. 12, pp. 3518-3524, 2010.

33. K. Boucart and A. M. Ionescu, "Double-gate tunnel fet with high-k gate dielectric," IEEE transactions on electron devices, vol. 54, no. 7, pp. 1725-1733, 2007.

34. M. Yadav, A. Bulusu, and S. Dasgupta, "Super-threshold semi analytical channel potential model for dg tunnel fet," Journal of Computational Electronics, vol. 14, no. 2, pp. 566-573, 2015.

35. M. Venkatesh and N. Balamurugan, "Influence of threshold voltage performance analysis on dual halo gate stacked triple material dual gate tfet for ultra low power applications," Silicon, vol. 13, no. 1, pp. 275-287, 2021. 